\title{
Efficacy and safety of Sijunzi Decoction for chronic fatigue syndrome with spleen deficiency pattern: study protocol for a randomized, double-blind, placebo-controlled trial
}

\author{
Liang Dai ${ }^{1}$, Wen-Jun Zhou ${ }^{1}$, Miao Wang ${ }^{2}$, Shi-Gao Zhou ${ }^{2}$, Guang Ji ${ }^{1}$ \\ ${ }^{1}$ Institute of Digestive Diseases, ${ }^{2}$ Department of Internal Medicine of Traditional Chinese Medicine, Longhua Hospital, Shanghai University of \\ Traditional Chinese Medicine, Shanghai 200032, China \\ Contributions: (I) Conception and design: L Dai; (II) Administrative support: SG Zhou, G Ji; (III) Provision of study materials or patients: M Wang; (IV) \\ Collection and assembly of data: M Wang; (V) Data analysis and interpretation: WJ Zhou; (VI) Manuscript writing: All authors; (VII) Final approval \\ of manuscript: All authors. \\ Correspondence to: Prof. Guang Ji. Institute of Digestive Diseases, Longhua Hospital, Shanghai University of Traditional Chinese Medicine, 725 South \\ Wanping Road, Xuhui District, Shanghai 200032, China. Email: jiliver@vip.sina.com.
}

Background: Chronic fatigue syndrome (CFS), which is characterized by severe and disabling fatigue, has become an extensively concerned medical disorder in clinical practice. Due to the unclear etiology, current treatments are symptomatic or need assistance from psychology and kinesiology. Under the immature conditions in China, many patients would seek help from traditional Chinese medicine (TCM), in which Chinese herbal medicine (CHM) is one of the main interventions. Sijunzi Decoction (SJZD) is a classical formula and has been utilized in improving fatigue symptoms for a long time. However, lack of rigorouslydesigned randomized controlled trial limits its application and generalization in CFS management. Hence, we design this clinical trial to assess the effectiveness and safety of SJZD for CFS.

Methods: This is a single-center, randomized, double-blind, placebo-controlled trial. Two hundred and twelve patients with CFS will be recruited from public and equally allocated to SJZD group and placebo group. Based on the general education, these two groups will receive corresponding drugs twice a day for consecutive 2 months. The follow-up period will be 1 month. The primary outcome will be the change of Chalder fatigue scoring after treatment. Secondary outcomes include the short form-36 physical function subscale (SF36-PF), spleen deficiency rating scale, quality of life and self-rated clinical global impression (CGI) scales.

Discussion: The four ingredients of SJZD are Renshen (Radix Ginseng), Baizhu (Rbizoma Atractylodis Macrocephalae), Fulin (Poria) and Zhigancao (Radix Glycyrrbizae Preparata), which show potential to alleviate CFS on the foundation of available studies. The results of this trial will provide high-quality clinical evidence for the application of SJZD, and hope to further support a new TCM choice in CFS treatment.

Trial registration: ISRCTN23930966 (ISRCTN registry, registered on 28th May, 2019).

Keywords: Chronic fatigue syndrome (CFS); traditional Chinese medicine (TCM); Sijunzi Decoction (SJZD); randomized controlled trial

Submitted Jun 24, 2019. Accepted for publication Sep 10, 2019.

doi: $10.21037 /$ atm.2019.09.136

View this article at: http://dx.doi.org/10.21037/atm.2019.09.136 


\section{Introduction}

Chronic fatigue syndrome (CFS) is characterized by prolonged and disabling fatigue ( $>6$ months), as well other nonspecific somatic symptoms (1). Epidemiological information of CFS is limited. Available studies reported the prevalence of CFS fluctuated from $0.23 \%$ to $2.6 \%$ (2-4). Although not life-threatening, CFS would severely impact patients' quality of life and lead to tremendous social burden $(5,6)$.

Due to the unclear understanding of CFS pathophysiology, current interventions focus on symptomatic treatment and psychological behavior therapies (7). Based on current trials, cognitive behavioral therapy and graded exercise therapy possess relatively high-quality evidences. However, due to the conventional doctor-visiting custom and psychological barrier in Chinese population $(8,9)$, these two therapies cannot get comprehensive application. In addition, existed trials related to chemical agents did not get satisfactory results (10-13). Therefore, many Chinese patients would seek help from traditional Chinese medicine (TCM), in which Chinese herbal medicine (CHM) is the main form.

According to the TCM concept, spleen governs energy metabolism. Spleen deficiency would lead to reduced energy, which is a manifestation of CFS (14). Therefore, we choose "spleen deficiency" as the accompanying TCM pattern. Sijunzi Decoction (SJZD), which was served as the basic formula treating spleen deficiency, has been utilized for fatigue since Tang Dynasty (15). The four ingredients, namely Renshen (Radix Ginseng), Baizhu (Rhizoma Atractylodis Macrocephalae), Fulin (Poria) and Zhigancao (Radix Glycyrrbizae Preparata), showed certain pharmacological effects in energy metabolism, such as increasing adenosine triphosphate (ATP) level, regulating activity of glucokinase and phosphoglycerate kinase, regulating lipid synthesis, and stimulating mitochondrial function (16-21). However, there still lack of high-quality clinical evidence related to its application. Hence, we design this rigorous randomized, double-blind, clinical trial, to evaluate the actual effectiveness and safety of SJZD using placebo as comparator, and thus provide preliminary support for its application in CFS management.

\section{Methods and design}

\section{Study objectives}

This clinical trial aims to assess the efficacy and safety of SJZD for CFS patients.

\section{Study design and setting}

This trial is designed as a single-center, double-blind, placebo-controlled trial with two parallel groups to evaluate the effect of SJZD. The protocol was developed based on Standard Protocol Items: Recommendations for Interventional Trials (SPIRIT) 2013 statement and TCM extension (22-24). The SPIRIT checklist is presented in Supplementary (Table S1). A total of 212 CFS patients will be recruited. After inform consent acquisition and eligibility confirmation, participants will be randomly allocated to SJZD group and placebo group based on the ratio of 1:1. On the foundation of general health education, participants will go through a 2 -month treatment period receiving either SJZD or placebo, and then a 1-month follow-up period. The participant flowchart is shown in Figure 1, and the participant timeline is given in Table 1 .

Four visits will be arranged for each participant, namely baseline (visit 1), 1 month (visit 2), 2 months (visit 3) and 3 months (visit 4). At visit 1, clinical investigator will confirm participant's qualification, document general information and then give the first-month medications. Participants will be required to come back at visit 2 for midterm clinical evaluation and second-month medications. Visit 3 is set for the end of the treatment period, and only clinical evaluation will be arranged. Afterwards, visit 4 is set for evaluating the continued effect.

The study protocol (version: PZYH-DL-1.1) was approved by Medical Ethics Committee of Longhua Hospital Affiliated to Shanghai University of TCM (approval number: 2019LCSY020), and was registered in the ISRCTN registry (ISRCTN23930966). If there will be any changes in study design, Ethics Committee would be informed immediately. Informed consent will be obtained from each patient.

\section{Participants}

This clinical trial will be conducted at the Longhua Hospital Affiliated to Shanghai University of TCM. All participants will be recruited from the public through the outpatient clinic.

\section{Diagnostic criteria}

The diagnosis of CFS will be confirmed based on the Centers for Disease Control and Prevention (CDC) criteria (1). To be specific, individuals should have severe fatigue for longer than 6 months, and possess at least four of the following symptoms: (I) headache of new type, 


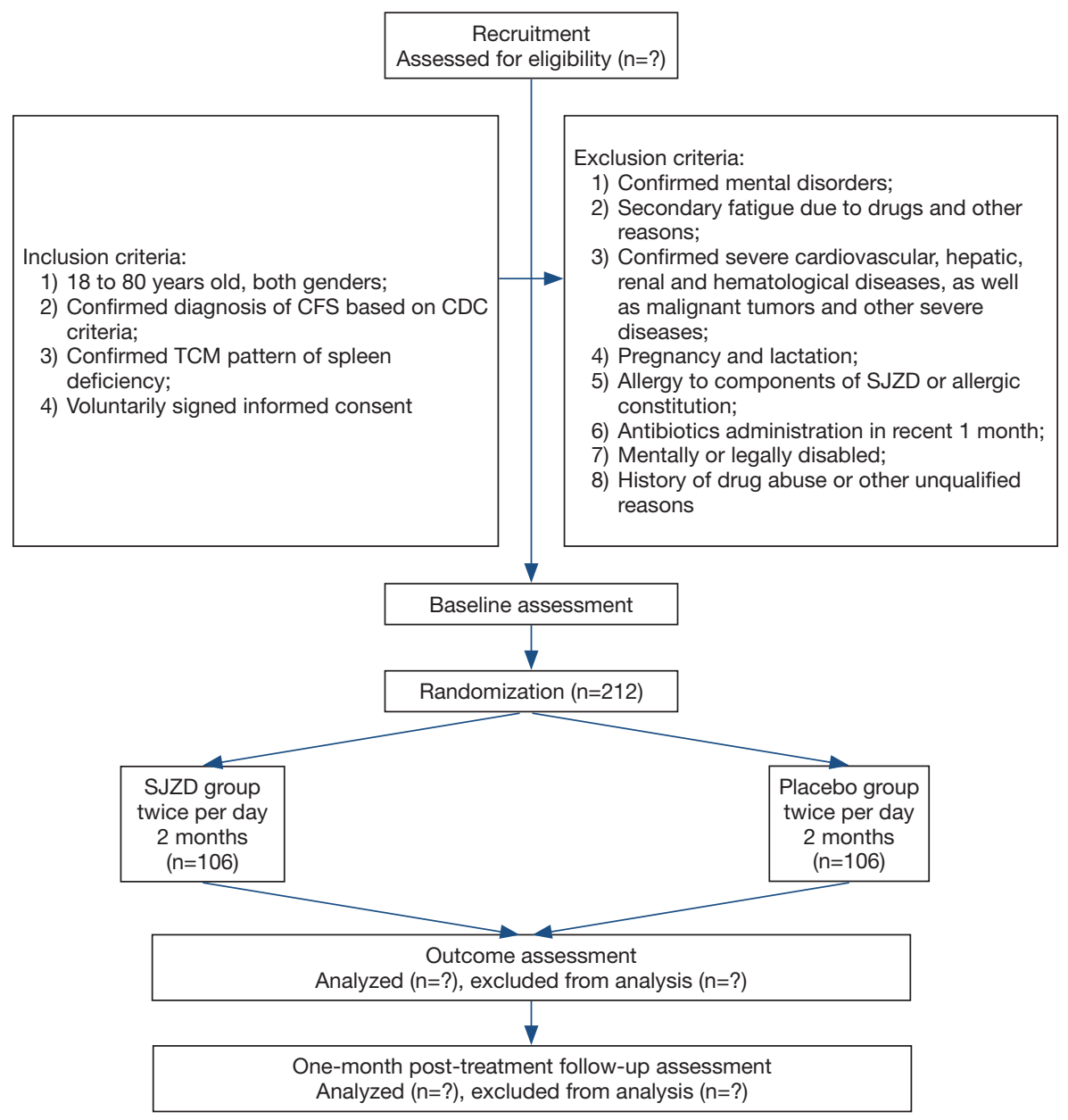

Figure 1 Participant flowchart. CDC, Centers for Disease Control and Prevention; SJZD, Sijunzi Decoction.

pattern, or severity; (II) multijoint pain without swelling or erythema; (III) muscle pain; (IV) postexertional malaise for longer than 24 hours; (V) significant impairment in shortterm memory or concentration; (VI) sore throat; (VII) tender lymph nodes; (VIII) unrefreshing sleep.

The pattern differentiation criteria for spleen deficiency refers to previous study and related guidelines (25-27). In brief, ten symptoms will be evaluated by patients themselves using 0-100 grading scale, namely (I) laziness to speak; (II) easy perspiration; (III) tasteless; (IV) loose stool; (V) increased saliva; (VI) gingival bleeding; (VII) cold limbs; (VIII) insomnia; (IX) easy getting cold; (X) change of diet habits. Higher scoring indicates higher severity. Each symptom has its own weighting factor. Clinical investigators with more than 5 -year experience will be responsible for scale inquiry. Spleen deficiency is defined as the accumulative scores of all symptoms above 20 . The detailed rating scale is shown in Table 2 .

\section{Inclusion criteria}

Participants who meet all of the following criteria could be included: (I) 18 to 80 years old, both genders; (II) confirmed diagnosis of CFS based on CDC criteria; (III) confirmed TCM pattern of spleen deficiency; (IV) voluntarily signed informed consent.

\section{Exclusion criteria}

Participants who meet any of the following criteria will be excluded: (I) confirmed mental disorders; (II) secondary fatigue due to drugs and other reasons; (III) confirmed severe cardiovascular, hepatic, renal and hematological diseases, as well malignant tumors and other severe diseases; (IV) pregnancy and lactation; (V) allergy to components of SJZD or allergic constitution; (VI) antibiotics administration in 
Table 1 Schedule of enrollment, interventions and assessments

\begin{tabular}{|c|c|c|c|c|c|}
\hline \multirow{2}{*}{$\begin{array}{l}\text { Study procedure } \\
\text { Timepoint }\end{array}$} & \multicolumn{5}{|c|}{ Study period } \\
\hline & Enrolment & $\frac{\text { Allocation }}{0}$ & 1-month & 2-month & $\frac{\text { Close-out }}{\text { 3-month }}$ \\
\hline \multicolumn{6}{|l|}{ Enrolment } \\
\hline Eligibility screen & $\star$ & & & & \\
\hline Allocation & & $\star$ & & & \\
\hline \multicolumn{6}{|l|}{ Interventions } \\
\hline Sijunzi Decoction & & & $\star$ & $\star$ & \\
\hline Placebo & & & $\star$ & $\star$ & \\
\hline Blood routine test & & $\star$ & & $\star$ & \\
\hline Liver and kidney function & & $\star$ & & $\star$ & \\
\hline 12-lead electrocardiogram & & $\star$ & & $\star$ & \\
\hline Chalder fatigue questionnaire & & $\star$ & $\star$ & $\star$ & $\star$ \\
\hline SF36-PF & & $\star$ & $\star$ & $\star$ & $\star$ \\
\hline Euroqol Questionnaire & & $\star$ & $\star$ & $\star$ & $\star$ \\
\hline CGI & & & & $\star$ & $\star$ \\
\hline Spleen deficiency rating scale & & $\star$ & $\star$ & $\star$ & $\star$ \\
\hline
\end{tabular}

According to SPIRIT 2013 Statement: Defining Standard Protocol Items for Clinical Trials. CGI, clinical global impression; SF36-PF, short form-36 physical function subscale.

Table 2 Spleen deficiency rating scale

\begin{tabular}{lcc}
\hline Symptom & Weight (\%) & Score \\
\hline Laziness to speak & $20 \%$ \\
Easy perspiration & $5 \%$ \\
Tasteless & $10 \%$ \\
Loose stool & $20 \%$ \\
Increased saliva & $10 \%$ \\
Gingival bleeding & $5 \%$ \\
Cold limbs & $10 \%$ \\
Insomnia & $5 \%$ \\
Easy getting cold & $10 \%$ \\
Change of diet habits & $5 \%$ \\
Total & \\
\hline
\end{tabular}

recent 1 month; (VII) mentally or legally disabled; (VIII) history of drug abuse or other unqualified reasons.

\section{Interventions}

\section{General medical education}

During every visit, clinical investigators will inform participants in either group essential medical education information, such as taking breaks timely, regular physical exercise, keeping smooth state, and avoiding negative emotions. Symptomatic treatment for accompanying symptoms will also be allowed.

\section{CHM intervention}

SJZD is composed of Renshen (Radix Ginseng), Baizhu 
Table 3 Composition and action of Sijunzi Decoction

\begin{tabular}{|c|c|c|c|}
\hline Ingredients & Content & TCM action & Pharmacological action \\
\hline $\begin{array}{l}\text { Baizhu (Rhizoma } \\
\text { Atractylodis } \\
\text { Macrocephalae) }\end{array}$ & $9 \mathrm{~g}$ & $\begin{array}{l}\text { Tonifying qi and } \\
\text { strengthening spleen }\end{array}$ & $\begin{array}{l}\text { (I) Improving immune function; (II) strengthening body; (III) regulating } \\
\text { gastrointestinal motility; (IV) diuretic effect }\end{array}$ \\
\hline Fulin (Poria) & $9 \mathrm{~g}$ & $\begin{array}{l}\text { Strengthening spleen and } \\
\text { promoting diuresis }\end{array}$ & $\begin{array}{l}\text { (I) Increasing the level of potassium in myocardium; (II) liver protective } \\
\text { effect; (III) diuretic effect }\end{array}$ \\
\hline $\begin{array}{l}\text { Zhigancao (Radix } \\
\text { Glycyrrhizae Preparata) }\end{array}$ & $6 \mathrm{~g}$ & $\begin{array}{l}\text { Tonifying qi and spleen, } \\
\text { harmonizing herbal nature }\end{array}$ & $\begin{array}{l}\text { (I) Regulating immune function; (II) promoting brain function; (III) anti- } \\
\text { infection and anti-inflammation effects; (IV) antiarrhythmic effect }\end{array}$ \\
\hline
\end{tabular}

HPA, hypothalamic-pituitary-adrenal.

(Rhizoma Atractylodis Macrocephalae), Fulin (Poria) and Zhigancao (Radix Glycyrrbizae Preparata). The specific composition of one daily dosage and related actions are listed in Table 3. The placebo is the simulant granule, which the main components are starch and dextrin. In order to present comparable color, smell, taste and texture with SJZD, food colorants and flavoring agents are added. Both SJZD and placebo are prepared as soluble granule by a third company, which is qualified in CHM processing and preparation. The third pharmaceutical company will equally divide daily dose (SJZD or placebo) into two individual pack. Participants from either group will be required to dissolve one pack with $200 \mathrm{~mL}$ hot water and take the decoction orally twice daily for 2 months. Both SJZD and placebo will be packed in sealed medicine box. Only the serial number will be printed outside the package to ensure successful blinding.

\section{Outcomes}

Throughout the study, participants will be required to complete a series of questionnaires. Due to the unclear pathophysiology mechanism of CFS, no efficacy related biochemical test will be arranged. However, blood sample and stool sample will still be collected at baseline and treatment endpoint. for future deep mechanism exploration. At baseline, clinical investigators will obtain inform consent and document general information such as age, gender, height, weight and medical history. For safety concern, routine blood test, liver and kidney function test and 12lead electrocardiogram (ECG) will be conducted at baseline and treatment endpoint (2 months). Additionally, vital signs and adverse events (AEs) will be monitored during the whole study.

The primary outcome will be the change of Chalder fatigue questionnaire after treatment. The Chalder fatigue questionnaire, which contains 11 questions in total, is a classic questionnaire measuring fatigue and has been widely used in previous CFS clinical trials (28-30). Each question includes four options, namely "better than usual", "no more than usual", "worse than usual" and "much worse than usual", indicating Likert scoring 0, 1, 2 and 3, respectively. The cumulative score ranges from 0 to 33 , and lowest score is least fatigue.

Secondary outcomes include the short form-36 physical function subscale (SF36-PF), spleen deficiency rating scale, quality of life [assessed by the Euroqol Questionnaire (EQ5D-5L)] and self-rated clinical global impression scales [including overall health (CGI-health) and CFS (CGICFS)]. SF36-PF is a valid and commonly-used assessment tool in evaluating the impact of CFS on patient's daily life $(29,30)$. The score ranges from 0 to 100 , and the highest score indicates best function. In this trial, we introduce the concept of TCM spleen deficiency, thus the spleen deficiency rating scale will be used for quantificationally assessing the change of TCM pattern. EQ-5D-5L is a modified measure of quality of life, and has also been applied in related studies (30-33). It contains an EQ-5D descriptive system focusing five dimensions, and an EQVisual Analogue Scale (VAS) scoring the overall health status. CGI scale contains seven options, which could be categorized into three classes: negative change (very much worse or much worse), no obvious change (a little worse, no change, or a little better), and positive change (much better 
or very much better). This is a self-rated tool assisting participant to recognize the therapeutic change from baseline (30).

Safety outcomes include documentation of AEs, physical examination, vital signs, and the relevant laboratory examination mentioned above. If any AEs happen, clinical investigators should record the onset time, related symptoms and signs, duration, abnormal laboratory indexes, intervention and prognosis. Then, the relationship with experimental drugs will be evaluated based on these data. The detailed arrangements of every outcome are presented in Table 1.

\section{Randomization and blinding}

Eligible participants will be randomly and equally allocated in two SJZD group or placebo group based on randomization sequence table generated by SPSS 22.0 for Windows. A specific statistical researcher who does not participate in the clinical trial will be responsible for generating the randomization sequence and distribute the number to the experimental products. Afterwards, clinical investigators will randomly assign the drug based on enrollment order. Intentionally selecting is strictly forbidden. The blinding base containing the randomization sequence, parameters of sequence, and treatments assignment is sealed and reserved by the principal investigator. Participants and clinical investigators will only notice the individual random number. At the last visit, participants and clinical investigators will complete a questionnaire about treatments assignment to verify the success of blinding. Emergency letters containing random number and treatment assignment will also be prepared by the specific statistical researcher. Only emergency when the actual intervention is necessary for further management could allow code breaks.

\section{Sample size calculation}

The sample size estimation is based on the primary outcome. To our knowledge, this is the first clinical trial assessing effect of a CHM formula on CFS with a specific TCM pattern, no previous related data could be found. Therefore, after discussion with clinical specialists, we assume that SJZD could reduce 3 point in Chalder fatigue scale, while placebo could reduce 0.5 point. In addition, based on previous study, the CFS patients had a mean fatigue score of $24.4 \pm 5.8$ (34). Therefore, the sample size of each group could be calculated by using the following formula (35):

$$
\mathrm{n}=\frac{\left(u_{\alpha}+u_{\beta}\right)^{2}(1+1 / k) \sigma^{2}}{\delta^{2}}
$$

We set type $I$ error $\alpha=0.05$ and a power of $90 \%(\beta=0.10)$. Two equal groups are designed, hence $\mathrm{k}=1$. As mentioned above, $\sigma$ will be 5.8, and $\delta$ will be 2.5. Ninety-two subjects are needed in one single group. Considering a $15 \%$ dropout, a total of 212 subjects are determined.

\section{Statistical analysis}

All outcomes will be analyzed based on the intention-totreat (ITT) principal. The missing value will be filled up by last-observation-carried-forward method. SPSS 22.0 for Windows will be used, and the statistical significance is defined as two-tailed $\mathrm{P}<0.05$. For the quantitative data, mean, standard deviation (SD), minimum, maximum and median will be reported. The paired quantitative data will also present the mean and SD of difference. For the enumerative data, frequency and corresponding percentage will be given. For intra-group comparisons during the study, paired $t$ test or Wilcoxon signed rank test will be used for quantitative indexes, and chi-squared test will be utilized for qualitative indexes. For comparisons between groups, twosample $t$-test or suitable non-parameter methods will be applied for quantitative indexes, and chi-squared test will be utilized for qualitative indexes. Clinical effectiveness should be confirmed after evaluating the clinical significance.

\section{Data collection and quality control}

This is a 3-month clinical trial. During the whole study, participants will be required to take assigned medication for consecutive 2 months, visit clinical investigator at four timepoints, complete some questionnaires and provide two rounds biomedical samples. The original data will be recorded comprehensively in case report form (CRF). Nobody except for principal investigators and clinical investigators is qualified to review the documents. The original records will be preserved at least 5 years after study completion.

In order to enhance the quality and stability of this clinical trial, clinicians with more than 5-year experience are eligible to be clinical investigators. Before the recruitment, all related researchers will undergo several training courses to guarantee the comprehensive understanding of study 
protocol, questionnaire evaluation and research process. Participants' compliance is a vital factor of the success of clinical trial. Hence, the following principles will be executed throughout the study. Firstly, clinical investigators should strictly comply with the principle of informed consent and assist participants to understand the possible benefits and risks. Secondly, clinical investigators should require participants to bring the used packs back in visit 2 and 3 , then to examine the administration situation. Thirdly, investigators may contact participants through phone and texts to remind the following visit three days in advance. For participants who present poor compliance, investigators should ask possible reasons and encourage them to complete the study.

\section{Discussion}

CFS has become a widespread problem worldwide. However, the underlying etiology of CFS is still in the mist. Early researches reported that post viral infections, such as Epstein-Barr virus (EBV), enterovirus and xenotropic murine leukemia virus-related virus (XMRV), could be noticed in CFS patients (36-38). However, the evidences are not consolidated $(39,40)$. Abnormal immune function is also a potential etiological factor of CFS, due to the findings which CFS patients manifested imbalanced natural killer (NK) cells and interleukin (IL) $(41,42)$. Similarly, some researches also showed conflicted results $(43,44)$. In addition, CFS patients may present lower cortisol level (45), certain genetic susceptibility (46), and negative psychological mood (47). Hence, based on available researches, the onset of CFS may be related to immune system, neuroendocrine system, genetics and biopsychosocial model.

To our knowledge, this is the first randomized controlled clinical trial evaluating a CHM formula for CFS treatment. SJZD is a classical prescription in clinical practice, and for the underlying pathogenesis mentioned above, a variety of pharmacological studies have illustrated the potential suitability of SJZD in CFS management. For instance, recent study showed that modified SJZD could regulate immune disorders in chronic atrophic gastritis patients with fatigue and tiredness symptom (48). And in a special immunosuppression rat model, SJZD could also improve immune function by regulate janus kinase (JAK)signal transducer and activator of transcription (STAT) signal pathway (49). Besides, in over fatigue and irregular diet rat model, SJZD could improve hypothalamic- pituitary-adrenal (HPA) axis function by elevating the level of adrenocorticotropic hormone (ACTH) and corticosterone (50). Therefore, on the foundation of previous researches, it is reasonable to choose SJZD as the intervention.

In this clinical trial, we choose generally-accepted scale as our outcomes. Nevertheless, we will still collect participants' blood and stool samples for potential mechanism exploration. Gut microbiota is considered to be an important site of energy metabolism (51). Hence, we curiously wonder whether the effect brought by SJZD, if any, involves the alteration of gut microbiota. This is also an innovative attempt among similar type trials.

In conclusion, this protocol is strictly developed according to the requirements of SPIRIT statement and corresponding extension. The results generated from this rigorous design would have high reliability, and provide preliminary evidence of SJZD in CFS management.

\section{Acknowledgments}

Funding: This work is supported by Shanghai Threeyear Action Plan for Accelerating the Development of Traditional Chinese Medicine [ZY(2018-2020)CCCX-2002-01].

\section{Footnote}

Conflicts of Interest: The authors have no conflicts of interest to declare.

Ethical Statement: The authors are accountable for all aspects of the work in ensuring that questions related to the accuracy or integrity of any part of the work are appropriately investigated and resolved. The study protocol was approved by Medical Ethics Committee of Longhua Hospital Affiliated to Shanghai University of TCM (Approval Number: 2019LCSY020). Informed consent will be obtained from each patient.

Disclaimer: The funding body had no role in study design, data collection and analysis, decision to publish, or preparation of the manuscript.

Data sharing plan: The datasets generated during and/or analyzed during the current study will be available upon request from principle investigator. Individual participant data that underlie the results reported in final report will 
become available for share, after deidentification. Data will be available beginning 6 months and ending 36 months following the final report publication. Researchers should provide a methodologically sound proposal to get data access. And researchers will only be allowed to use the data for the prescribed aims documented in the proposal. To gain access, data requestors will need to sign a data access agreement. Further inform consent may be considered according to the study aims. The shared data will only be allowed to be used by the applicant for scientific studies. No commercial activities are allowed.

\section{References}

1. Fukuda K, Straus SE, Hickie I, et al. The chronic fatigue syndrome: a comprehensive approach to its definition and study. International Chronic Fatigue Syndrome Study Group. Ann Intern Med 1994;121:953-9.

2. Wessely S, Chalder T, Hirsch S, et al. The prevalence and morbidity of chronic fatigue and chronic fatigue syndrome: a prospective primary care study. Am J Public Health 1997;87:1449-55.

3. Jason LA, Richman JA, Rademaker AW, et al. A community-based study of chronic fatigue syndrome. Arch Intern Med 1999;159:2129-37.

4. Reyes M, Nisenbaum R, Hoaglin DC, et al. Prevalence and incidence of chronic fatigue syndrome in Wichita, Kansas. Arch Intern Med 2003;163:1530-6.

5. Cairns R, Hotopf M. A systematic review describing the prognosis of chronic fatigue syndrome. Occup Med (Lond) 2005;55:20-31.

6. Reynolds KJ, Vernon SD, Bouchery E, et al. The economic impact of chronic fatigue syndrome. Cost Eff Resour Alloc 2004;2:4.

7. Cleare AJ, Reid S, Chalder T, et al. Chronic fatigue syndrome. BMJ Clin Evid 2015;2015.

8. Chen J. Some People May Need it, But Not Me, Not Now: Seeking Professional Help for Mental Health Problems in Urban China. Transcult Psychiatry 2018;55:754-74.

9. Sun KS, Lam TP, Lam KF, et al. Barriers and facilitators for psychiatrists in managing mental health patients in Hong Kong-Impact of Chinese culture and health system. Asia Pac Psychiatry 2018. doi: 10.1111/appy.12279.

10. Cleare AJ, Miell J, Heap E, et al. Hypothalamo-pituitaryadrenal axis dysfunction in chronic fatigue syndrome, and the effects of low-dose hydrocortisone therapy. J Clin Endocrinol Metab 2001;86:3545-54.
11. Blockmans D, Persoons P, Van Houdenhove B, et al. Combination therapy with hydrocortisone and fludrocortisone does not improve symptoms in chronic fatigue syndrome: a randomized, placebo-controlled, double-blind, crossover study. Am J Med 2003;114:736-41.

12. Kogelnik AM, Loomis K, Hoegh-Petersen M, et al. Use of valganciclovir in patients with elevated antibody titers against Human Herpesvirus-6 (HHV-6) and EpsteinBarr Virus (EBV) who were experiencing central nervous system dysfunction including long-standing fatigue. J Clin Virol 2006;37 Suppl 1:S33-8.

13. Chambers D, Bagnall AM, Hempel S, et al. Interventions for the treatment, management and rehabilitation of patients with chronic fatigue syndrome/myalgic encephalomyelitis: an updated systematic review. J R Soc Med 2006;99:506-20.

14. Deng T, Guo Z. Diagnosis in Chinese Medicine. Shanghai: Shanghai Science and Technology Press, 1984.

15. Xu J, Wang M. Formulas of Chinese Medicine. Shanghai: Shanghai Science and Technology Press, 1984.

16. Li XT, Chen R, Jin LM, et al. Regulation on energy metabolism and protection on mitochondria of Panax ginseng polysaccharide. Am J Chin Med 2009;37:1139-52.

17. Wang JR, Zhou H, Yi XQ, et al. Total ginsenosides of Radix Ginseng modulates tricarboxylic acid cycle protein expression to enhance cardiac energy metabolism in ischemic rat heart tissues. Molecules 2012;17:12746-57.

18. Song MY, Kang SY, Oh TW, et al. The Roots of Atractylodes macrocephala Koidzumi Enhanced Glucose and Lipid Metabolism in C2C12 Myotubes via Mitochondrial Regulation. Evid Based Complement Alternat Med 2015;2015:643654.

19. Song MY, Lim SK, Wang JH, et al. The Root of Atractylodes macrocephala Koidzumi Prevents Obesity and Glucose Intolerance and Increases Energy Metabolism in Mice. Int J Mol Sci 2018. doi: 10.3390/ijms19010278.

20. Han XY, Wang YN, Dou DQ. Regulatory effects of Poria on substance and energy metabolism in cold-deficiency syndrome compared with heat-deficiency syndrome in rats. Chin J Nat Med 2018;16:936-45.

21. Madak-Erdogan Z, Gong P, Zhao YC, et al. Dietary licorice root supplementation reduces diet-induced weight gain, lipid deposition, and hepatic steatosis in ovariectomized mice without stimulating reproductive tissues and mammary gland. Mol Nutr Food Res 2016;60:369-80.

22. Dai L, Cheng CW, Tian R, et al. Standard Protocol Items for Clinical Trials with Traditional Chinese Medicine 
2018: Recommendations, Explanation and Elaboration (SPIRIT-TCM Extension 2018). Chin J Integr Med 2019;25:71-9.

23. Chan AW, Tetzlaff JM, Altman DG, et al. SPIRIT 2013 statement: defining standard protocol items for clinical trials. Ann Intern Med 2013;158:200-7.

24. Chan AW, Tetzlaff JM, Gotzsche PC, et al. SPIRIT 2013 explanation and elaboration: guidance for protocols of clinical trials. BMJ 2013;346:e7586.

25. Zheng X. Guiding principles for clinical research of new TCM drugs (on trial). Beijing: China Medical Science Press, 2002.

26. Lin J. Development and assessment of PRO scale of qi deficiency of spleen. Fujian University of Traditional Chinese Medicine, 2013.

27. Zhang SS, Hu L, Li RL. Expert consensus on diagosis and treatment of spleen deficiency with Chinese Medicine. Journal of Traditional Chinese Medicine 2017;58:1525-30.

28. Chalder T, Berelowitz G, Pawlikowska T, et al. Development of a fatigue scale. J Psychosom Res 1993;37:147-53.

29. White PD, Goldsmith KA, Johnson AL, et al. Comparison of adaptive pacing therapy, cognitive behaviour therapy, graded exercise therapy, and specialist medical care for chronic fatigue syndrome (PACE): a randomised trial. Lancet 2011;377:823-36.

30. Clark LV, Pesola F, Thomas JM, et al. Guided graded exercise self-help plus specialist medical care versus specialist medical care alone for chronic fatigue syndrome (GETSET): a pragmatic randomised controlled trial. Lancet 2017;390:363-73.

31. Herdman M, Gudex C, Lloyd A, et al. Development and preliminary testing of the new five-level version of EQ-5D (EQ-5D-5L). Qual Life Res 2011;20:1727-36.

32. Janssen MF, Pickard AS, Golicki D, et al. Measurement properties of the EQ-5D-5L compared to the EQ-5D-3L across eight patient groups: a multi-country study. Qual Life Res 2013;22:1717-27.

33. Kim JE, Seo BK, Choi JB, et al. Acupuncture for chronic fatigue syndrome and idiopathic chronic fatigue: a multicenter, nonblinded, randomized controlled trial. Trials 2015;16:314.

34. Cella M, Chalder T. Measuring fatigue in clinical and community settings. J Psychosom Res 2010;69:17-22.

35. Wang J. Clinical epidemiology_design, measure and appraise of clinical researches. 4th ed. Shanghai: Shanghai Science and Technology Press, 2014.

36. Straus SE, Tosato G, Armstrong G, et al. Persisting illness and fatigue in adults with evidence of Epstein-Barr virus infection. Ann Intern Med 1985;102:7-16.

37. Chia JK. The role of enterovirus in chronic fatigue syndrome. J Clin Pathol 2005;58:1126-32.

38. Lombardi VC, Ruscetti FW, Das Gupta J, et al. Detection of an infectious retrovirus, XMRV, in blood cells of patients with chronic fatigue syndrome. Science 2009;326:585-9. Erratum in: Science. 2011 Oct 14;334(6053):176. Retraction in: Alberts B. Science. 2011 Dec 23;334(6063):1636.

39. Mawle AC, Nisenbaum R, Dobbins JG, et al. Seroepidemiology of chronic fatigue syndrome: a casecontrol study. Clin Infect Dis 1995;21:1386-9.

40. van Kuppeveld FJ, de Jong AS, Lanke KH, et al. Prevalence of xenotropic murine leukaemia virus-related virus in patients with chronic fatigue syndrome in the Netherlands: retrospective analysis of samples from an established cohort. BMJ 2010;340:c1018.

41. Brenu EW, Staines DR, Baskurt OK, et al. Immune and hemorheological changes in chronic fatigue syndrome. J Transl Med 2010;8:1.

42. Fletcher MA, Zeng XR, Barnes Z, et al. Plasma cytokines in women with chronic fatigue syndrome. J Transl Med 2009;7:96.

43. Vernon SD, Reeves WC. Evaluation of autoantibodies to common and neuronal cell antigens in Chronic Fatigue Syndrome. J Autoimmune Dis 2005;2:5.

44. Lorusso L, Mikhaylova SV, Capelli E, et al. Immunological aspects of chronic fatigue syndrome. Autoimmun Rev 2009;8:287-91.

45. Cevik R, Gur A, Acar S, et al. Hypothalamic-pituitarygonadal axis hormones and cortisol in both menstrual phases of women with chronic fatigue syndrome and effect of depressive mood on these hormones. BMC Musculoskelet Disord 2004;5:47.

46. Whistler T, Jones JF, Unger ER, et al. Exercise responsive genes measured in peripheral blood of women with chronic fatigue syndrome and matched control subjects. BMC Physiol 2005;5:5.

47. Chalder T, Godfrey E, Ridsdale L, et al. Predictors of outcome in a fatigued population in primary care following a randomized controlled trial. Psychol Med 2003;33:283-7.

48. Tian G, Wu C, Li J, et al. Network pharmacology based investigation into the effect and mechanism of Modified Sijunzi Decoction against the subtypes of chronic atrophic gastritis. Pharmacol Res 2019;144:158-66.

49. Xiong B, Qian H. Effects of Sijunzi decoction and 
Yupingfeng powder on expression of janus kinase-signal transducer and activator of transcription signal pathway in the brain of spleen-deficiency model rats. J Tradit Chin Med 2013;33:78-84.

50. Wang H. Comparison of biological effects of Sijunzitang (SJZT), Chaishusijuntang (CSSJT) and Chaihushugansan

Cite this article as: Dai L, Zhou WJ, Wang M, Zhou SG, Ji G. Efficacy and safety of Sijunzi Decoction for chronic fatigue syndrome with spleen deficiency pattern: study protocol for a randomized, double-blind, placebo-controlled trial. Ann Transl Med 2019;7(20):587. doi: 10.21037/atm.2019.09.136
(CHSGS) on the animal model of the syndromes of spleen-qi deficiency [Medical Doctor]: Beijing University of Chinese Medicine, 2007.

51. Bauer PV, Hamr SC, Duca FA. Regulation of energy balance by a gut-brain axis and involvement of the gut microbiota. Cell Mol Life Sci 2016;73:737-55. 
Table S1 SPIRIT 2013 Checklist: recommended items to address in a clinical trial protocol and related documents ${ }^{*}$

\begin{tabular}{|c|c|c|c|}
\hline Section/item & Item No. & Description & Addressed on page number \\
\hline \multicolumn{4}{|l|}{ Administrative information } \\
\hline Title & 1 & Descriptive title identifying the study design, population, interventions, and, if applicable, trial acronym & 1 \\
\hline \multirow[t]{2}{*}{ Trial registration } & $2 a$ & Trial identifier and registry name. If not yet registered, name of intended registry & 1 \\
\hline & $2 \mathrm{~b}$ & All items from the World Health Organization Trial Registration Data Set & NA \\
\hline Protocol version & 3 & Date and version identifier & 2 \\
\hline Funding & 4 & Sources and types of financial, material, and other support & 9 \\
\hline \multirow[t]{4}{*}{ Roles and responsibilities } & $5 a$ & Names, affiliations, and roles of protocol contributors & 1 \\
\hline & $5 b$ & Name and contact information for the trial sponsor & 1 \\
\hline & $5 c$ & $\begin{array}{l}\text { Role of study sponsor and funders, if any, in study design; collection, management, analysis, and interpretation of data; writing of the report; and the decision to } \\
\text { submit the report for publication, including whether they will have ultimate authority over any of these activities }\end{array}$ & 9 \\
\hline & $5 \mathrm{~d}$ & $\begin{array}{l}\text { Composition, roles, and responsibilities of the coordinating centre, steering committee, endpoint adjudication committee, data management team, and other } \\
\text { individuals or groups overseeing the trial, if applicable [see Item } 21 \text { a for data monitoring committee (DMC)] }\end{array}$ & NA \\
\hline \multicolumn{4}{|l|}{ Introduction } \\
\hline \multirow[t]{2}{*}{ Background and rationale } & $6 a$ & $\begin{array}{l}\text { Description of research question and justification for undertaking the trial, including summary of relevant studies (published and unpublished) examining benefits } \\
\text { and harms for each intervention }\end{array}$ & 2 \\
\hline & $6 b$ & Explanation for choice of comparators & 2 \\
\hline Objectives & 7 & Specific objectives or hypotheses & 2 \\
\hline Trial design & 8 & $\begin{array}{l}\text { Description of trial design including type of trial (e.g., parallel group, crossover, factorial, single group), allocation ratio, and framework (e.g., superiority, } \\
\text { equivalence, noninferiority, exploratory) }\end{array}$ & 2 \\
\hline
\end{tabular}

Methods: participants, interventions, and outcomes

Study setting Description of study settings (e.g., community clinic, academic hospital) and list of countries where data will be collected. Reference to where list of study sites
can be obtained

Eligibility criteria

Interventions

10 Inclusion and exclusion criteria for participants. If applicable, eligibility criteria for study centres and individuals who will perform the interventions (e.g., surgeons, psychotherapists)

11a Interventions for each group with sufficient detail to allow replication, including how and when they will be administered

$11 \mathrm{~b}$ Criteria for discontinuing or modifying allocated interventions for a given trial participant (e.g., drug dose change in response to harms, participant request, or improving/worsening disease)

$11 \mathrm{C}$ Strategies to improve adherence to intervention protocols, and any procedures for monitoring adherence (e.g., drug tablet return, laboratory tests)

11d Relevant concomitant care and interventions that are permitted or prohibited during the tria

Outcomes

12 Primary, secondary, and other outcomes, including the specific measurement variable (e.g., systolic blood pressure), analysis metric (e.g., change from baseline, Primary, secondary, and other outcomes, including the specific measurement variable (e.g., systolic blood pressure), analysis metric (e.g., change from base
final value, time to event), method of aggregation (e.g., median, proportion), and time point for each outcome. Explanation of the clinical relevance of chosen final value, time to event), method of aggregation (e.g.,
efficacy and harm outcomes is strongly recommended

Participant timeline

Sample size

13 Time schedule of enrolment, interventions (including any run-ins and washouts), assessments, and visits for participants. A schematic diagram is highly recommended (see Figure)

Recruitment

14 Estimated number of participants needed to achieve study objectives and how it was determined, including clinical and statistical assumptions supporting any sample size calculation

Methods: assignment of interventions (for controlled trials)

Allocation:

Sequence generation

Allocation concealmen

mechanism

Implementation

Blinding (masking)

Methods: data collection, management, and analys

Data collection methods

18a Plans for assessment and collection of outcome, baseline, and other trial data, including any related processes to promote data quality (e.g., duplicate measurements, training of assessors) and a description of study instruments (e.g., questionnaires, laboratory tests) along with their reliability and validity, if know Reference to where data collection forms can be found, if not in the protocol

18b Plans to promote participant retention and complete follow-up, including list of any outcome data to be collected for participants who discontinue or deviate from intervention protocols

Data management

19 Plans for data entry, coding, security, and storage, including any related processes to promote data quality (e.g., double data entry; range checks for data values). Reference to where details of data management procedures can be found, if not in the protocol

Statistical methods

20a Statistical methods for analysing primary and secondary outcomes. Reference to where other details of the statistical analysis plan can be found, if not in the protocol

20b Methods for any additional analyses (e.g., subgroup and adjusted analyses)

20 Definition of analysis population relating to protocol non-adherence (e.g., as randomised analysis), and any statistical methods to handle missing data (e.g., multiple imputation

Methods: monitoring

Data monitoring

21a Composition of DMC; summary of its role and reporting structure; statement of whether it is independent from the sponsor and competing interests; and reference to where further details about its charter can be found, if not in the protocol. Alternatively, an explanation of why a DMC is not needed

21b Description of any interim analyses and stopping guidelines, including who will have access to these interim results and make the final decision to terminate the trial

Harms

22 Plans for collecting, assessing, reporting, and managing solicited and spontaneously reported adverse events and other unintended effects of trial interventions or trial conduct

Auditing

Ethics and dissemination

Research ethics approval

Protocol amendments

Consent or assent

Confidentiality

Declaration of interests

Access to data

Ancillary and post-trial care Dissemination policy

23 Frequency and procedures for auditing trial conduct, if any, and whether the process will be independent from investigators and the sponsor

24 Plans for seeking research ethics committee/institutional review board (REC/RB) approval

25 Plans for communicating important protocol modifications (e.g., changes to eligibility criteria, outcomes, analyses) to relevant parties (e.g. investigators, REC/ IRBs, trial participants, trial registries, jounals, regulators

26a Who will obtain informed consent or assent from potential trial participants or authorized surrogates, and how (see Item 32)

26 Additional consent provisions for collection and use of participant data and biological specimens in ancillary studies, if applicable

27 How personal information about potential and enrolled participants will be collected, shared, and maintained in order to protect confidentiality before, during, and after the trial

28 Financial and other competing interests for principal investigators for the overall trial and each study site

29 Statement of who will have access to the final trial dataset, and disclosure of contractual agreements that limit such access for investigators

30 Provisions, if any, for ancillary and post-trial care, and for compensation to those who suffer harm from trial participation

31a Plans for investigators and sponsor to communicate trial results to participants, healthcare professionals, the public, and other relevant groups (e.g., via publication, reporting in results databases, or other data sharing arrangements) including any publication restrictions

31b Authorship eligibility guidelines and any intended use of professional writers

$31 \mathrm{C}$ Plans, if any, for granting public access to the full protocol, participant-level dataset, and statistical code

Appendices

Informed consent materials Biological specimens

*, it is strongly recommended that this checklist be read in conjunction with the SPIRIT 2013 Explanation \& Elaboration for important clarficication on the items. Amendments to the protocol should be tracked and dated. The SPIRIT checklist is copyrighted by the SPIRIT Group under the Creative Commons "Attribution-NonCommercial-NoDerivs 3.0 Unported" license. NA, not available. 